\title{
1 Irrational risk aversion in ants is driven by perceptual mechanisms
}

2 Massimo De Agrò ${ }^{1,2, *}$, Daniel Grimwade ${ }^{1,3}$, and Tomer J. Czaczkes ${ }^{1}$

3 1) Animal Comparative Economics laboratory, Department of Zoology and Evolutionary Biology, University of 4 Regensburg, Germany

5 2) Department of General Psychology, University of Padova, Italy

6 3) Department of Zoology, University of Oxford, UK

7 * Corresponding author:

8 e-mail: massimo.deagro@phd.unipd.it

9 telephone: +390498276684

10 address: Via Venezia 12, Padova, Italy

12 Abstract

13 Animals must often decide between exploiting safe options or risky options with a chance for large 14 gains. While traditional optimal foraging theories assume rational energy maximisation, they fail to 15 fully describe animal behaviour. A logarithmic rather than linear perception of stimuli may shape preference, causing animals to make suboptimal choices. Budget-based rules have also been used to explain risk-preference, and the relative importance of these theories is debated. Eusocial insects represent a special case of risk sensitivity, as they must often make collective decisions based on resource evaluations from many individuals. Previously, colonies of the ant Lasius niger were found to be risk-neutral, but the risk preference of individual foragers was unknown. Here, we tested individual $L$. niger in a risk sensitivity paradigm. Ants were trained to associate a scent with $0.55 \mathrm{M}$ sucrose solution and another scent with an equal chance of either 0.1 and $1.0 \mathrm{M}$ sucrose. Preference was tested in a Y-maze. Ants were extremely risk averse, with $91 \%$ choosing the safe option. Even when the risky option offered on average more sucrose (0.8M) than the fixed option, 75\% preferred the latter. Based on the psychophysical Weber-Fechner law, we predicted that logarithmically balanced alternatives $(0.3 \mathrm{M}$ vs $0.1 \mathrm{M} / 0.9 \mathrm{M})$ would be perceived as having equal value. Our prediction was supported, with ants having no preference for either feeder (53\% chose the fixed option). Our results thus strongly support perceptual mechanisms driving riskaversion in ants, and demonstrate that the behaviour of individual foragers can be a very poor predictor of colony-level behaviour. 
bioRxiv preprint doi: https://doi.org/10.1101/620054; this version posted April 29, 2019. The copyright holder for this preprint (which was not certified by peer review) is the author/funder, who has granted bioRxiv a license to display the preprint in perpetuity. It is made available under aCC-BY-NC-ND 4.0 International license.

\section{Introduction}

Finding a good meal is not easy: the environment provides a broad variety of food sources, but individuals are not necessarily able to explore all of them before committing to one

34 (Mehlhorn et al., 2015). The food sources the organism inspects will often have different attributes, and options can be compared in order to choose the best one. This economic decision process is so crucial for organisms that the ability to compare options is found not only in animals, but even in non-neuronal organisms such as plants and slime-moulds (Dener et al., 2016; Reid et al., 2015, 2016). costs, on the basis that evolution should drive animals to have optimal behavioural strategies.

41 However, the optimal foraging theory framework (Pyke et al., 1977) fails to fully describe

42 behaviour - organisms do not always behave optimally. Extensive examples of violation of 43 optimality in animal species can be found, for example, in the literature about risk sensitivity. We

44 define risk as a situation in which the probabilities associated with an option (e.g. food source) are

45 known, but the exact value of it is not. Conversely, "uncertainty" is when not even the probabilities 46 of the various possible payoffs are known.

\section{Risk sensitivity theories - the budget rule}

Risk sensitivity studies were effectively inaugurated by Caraco et al. (1980). They studied

49 the preference of yellow-eyed juncos for different amount of seeds: one of the two alternatives

50 available to the birds was stable, presenting always the same, medium amount of food (safe

51 feeder), while the other one fluctuated in value, but had the same mean pay-out as the safe feeder

52 (risky feeder). The authors then, based on the preference of the animals, designed a utility

53 function (Becker et al., 1964), computing the perceived value (utility) for each number of seeds for

54 the animals. Yellow-eyed juncos presented a concave utility function (and so were risk averse) 
55 when in a high energy budget, whereas their utility function was convex (and so they were risk

56 prone) when in a low energy budget. This behaviour was soon formalized as the Energy Budget

57 Rule (Stephens, 1981). However, a growing body of work on risk sensitivity failed to provide

58 consistent empirical support for the budget rule (Kacelnik and Bateson, 1996; Kacelnik and El

59 Mouden, 2013). For this reason the budget rule has recently been reformulated by Lim et. al.

60 (2015). They argue that the classical budget rule is often misused in its binomial interpretation:

61 animals are either risk prone (when in a low energy budget) or risk averse (when in a high energy

62 budget). However, the optimum risk sensitivity in a given situation lies on a continuum, depending

63 on the remaining energy budget of the animal, even arriving at extreme conditions (very low

64 energy budget and very high energy budget) in which risk indifference arise again. Such a continuous interpretation of the budget rule may accommodate results considered as inconsistent the classical budget rule hypothesis (e.g. Hurly, 2003).

An alternative to prescriptive theories (based on optimality modelling) are descriptive

69 theories, which explain behaviours in terms of proximate mechanisms. If risk sensitivity arises as a

70 side-effect of the neural or cognitive architecture of an animal, or due to evolutionary constraints,

71 one need not attempt to fit this behaviour to fitness benefits. A striking pattern in risk preference

72 studies is that animals are often risk averse when risking amounts, but risk seeking when risking

73 delays (Kacelnik and Bateson, 1996). Animals (and humans) are also generally risk averse for

74 potential gains, but risk prone for potential losses (Kahneman and Tversky, 1979). These patterns

75 are elegantly explained by an understanding of how animals perceive the world, as described by

76 Psychophysics (Gescheider, 1976; Stevens, 2017; Tuzlukov, 2013). Stimulus strength has a

77 logarithmic relationship with perception, as formalized by the Weber-Fechner law (Fechner, 1860).

78 Thus, a constant feeder that always presents 5 seeds and a variable feeders presenting 
alternatively 1 or 9 seeds have the same average; however, 5 seeds are perceived as 5 times more

80 than 1 on a logarithmic curve, while 9 is not even twice as good as 5 . Thus, while the mathematical

81 average, and so the true energetic value, of the variable feeder is the same as the one of the safe

82 feeder, it's geometric average is lower. On logarithmic distributions, such as the Weber-Fechner

83 law by which animals perceive the world, the median is coincident with the geometrical average,

84 and is the measure that describes the overall perceived value of an option, as it is the middle point

85 between the two alternatives. Based on these insights, Kacelnik \& El Mouden (2013) developed

86 Scalar Utility Theory (SUT) to describe risk aversion behaviour. They point out that, based on the

87 Weber-Fechner law, the variance of the memory representation of a food value increases as the

88 value itself increases. For this reason, two options with identical mathematical average (means)

89 but different variances will have different medians, with the more variable option having a lower

90 one (see figure 6 from Kacelnik and El Mouden, 2013 for a complete explanation). However,

91 support for this descriptive theory is also mixed: Lim et al. (2015) argue that SUT has even weaker

92 support than the budget rule, with only 8 of the 35 studies reviewed by Kacelnic \& Bateson (1996)

93 finding complete risk aversion when risking potential resource gains. Shafir (2000) argued that it is

94 the strength of risk preference that is driven by perceptual mechanisms, while the direction is

95 driven by budget considerations, and could thus accommodate both risk seeking and risk aversion

96 in a manner consistent with logarithmic perception. However, Shafir's model can only account for

97 alternatives with the same mean value. Whether risk sensitivity is best understood in terms of

98 adaptation or constraints on perceptual mechanisms is thus still under debate.

Ants as a model for risk sensitivity

101 El Mouden, 2013). Among those, nectarivores have received particular scrutiny (Perez and

102 Waddington, 1996; Shafir, 2000). The majority of studies on nectarivores have been carried out on 
bioRxiv preprint doi: https://doi.org/10.1101/620054; this version posted April 29, 2019. The copyright holder for this preprint (which was not certified by peer review) is the author/funder, who has granted bioRxiv a license to display the preprint in perpetuity. It is made available under aCC-BY-NC-ND 4.0 International license.

103 bees. Results have, however, been unclear: bees have been observed to be risk indifferent

104 (Banschbach and Waddington, 1994; Fülöp and Menzel, 2000; Perez and Waddington, 1996), risk

105 averse (Shapiro, 2000; Waddington et al., 1981), to follow the budget rule (Cartar, 1991; Cartar and

106 Dill, 1990), or a mixture of those depending on risk variability (Dunlap et al., 2017; Mayack and

107 Naug, 2011; Shafir, 2000; Shafir et al., 1999). Bees and other eusocial insects represent a special

108 case for risk sensitivity. For eusocial insects with non-reproductive workers, the colony is the main

109 unit of selection and a colony can be considered a superorganisms (Boomsma and Gawne, 2018;

110 Hölldobler and Wilson, 2009). As such, the foraging successes of the individual workers are pooled.

111 This buffers colonies against short-term (negative) fluctuation coming from risky choices made by

112 individual foragers individuals. Colonies can also visit multiple food sources simultaneously,

113 allowing them to more efficiently exploit their environment (Czaczkes et al., 2015a; Devigne and

114 Detrain, 2005). Lastly, many eusocial insects can make collective foraging decisions, using

115 recruitment mechanisms to channel workers towards certain resources in the environment

116 (Detrain and Deneubourg, 2008; Gordon, 2019).

While research on risk preference and collective decision-making is extensive, these have

118 rarely been combined. Collective risk sensitivity has been explicitly studied in ants: Burns et al.

119 (2016) presented colonies of rock ants (Temnothorax albipennis) a fixed-quality mediocre nest and

120 a variable quality nest. Ants were allowed to explore (and hence evaluate) each nest and then

121 recruited nestmates, and colonies were found to be risk prone. On the other hand, Hübner \&

122 Czaczkes (2017) tested the risk sensitivity of black garden ant (Lasius niger) colonies to food values.

123 Each colony was presented with two feeders: a stable one, always presenting the same, medium

124 quality sucrose solution (0.55M), and a variable one, presenting alternatively (changing every 3

125 minutes) either low or high quality sucrose solution (0.1M-1.0M). Almost all trials showed a clear

126 collective decision for one of the two feeders (as is expected due to symmetry breaking in ants

127 collective decisions, see Beckers et al., 1990, 1993; Czaczkes et al., 2015b; Price et al., 2016), but 
bioRxiv preprint doi: https://doi.org/10.1101/620054; this version posted April 29, 2019. The copyright holder for this preprint (which was not certified by peer review) is the author/funder, who has granted bioRxiv a license to display the preprint in perpetuity. It is made available under aCC-BY-NC-ND 4.0 International license.

128 overall colonies were risk-indifferent: half the colonies chose the safe feeder, and half chose the

129 risky one. This is surprising, as positive feedback from the initially best food source should have

130 resulted in symmetry breaking and a collective choice for that feeder (Beckers et al., 1993;

131 Czaczkes et al., 2015b; Detrain and Deneubourg, 2008; Price et al., 2016).

This work aimed to explore individual risk preference in individual Lasius niger ant foragers.

133 Although their collective behaviour appears to be rational, individual workers may not be (Sasaki

134 and Pratt, 2011). They could be victims to the same perceptual constrains discussed above and be

135 strongly influenced by expectations, causing disappointment for some food alternatives, triggering 136 risk sensitivity.

137 Materials and Methods

138 Subjects

8 queenless Lasius niger colony fragments of around 1000 ants were used in the

experiment. Each fragment was collected from a different wild colony on the University of

141 Regensburg campus. Colonies fragments forage, deposit pheromone and learn well (Evison et al.,

142 2008; Oberhauser et al., 2018). Each fragment was housed in a transparent plastic box

$143(30 \times 20 \times 40 \mathrm{~cm})$, with a layer of plaster on the bottom. A circular plaster nest, $14 \mathrm{~cm}$ in diameter and

$1442 \mathrm{~cm}$ thick, was also provided. The colonies were kept at room temperature $\left(21-25 \mathrm{c}^{\circ}\right)$ and

145 humidity (45-55\%), on 12:12 light:dark cycle.

Each colony was fed $0.5 \mathrm{~mol}$ sucrose solution ad libitum, and deprived of food 4 days prior

147 each test. Water was provided ad libitum and was always present.

148 Experiment 1 - Risk preference between options of equal absolute value 
bioRxiv preprint doi: https://doi.org/10.1101/620054; this version posted April 29, 2019. The copyright holder for this preprint (which was not certified by peer review) is the author/funder, who has granted bioRxiv a license to display the preprint in perpetuity. It is made available under aCC-BY-NC-ND 4.0 International license.

151 moderate value ( $0.55 \mathrm{M}$ sucrose, the 'safe' option) and one providing a fluctuating value, either

152 high or low (0.1M or $1.0 \mathrm{M}$, the 'risky' option). This was achieved by teaching each individual ant to

153 associate each feeder type (risky or safe) with a different odour, and then testing their preference

154 in a Y-maze. Preliminary tests (see ESM1) and previous work (Czaczkes et al., 2018b, 2018c) shows

155 that L. niger foragers learn quickly (within 3 visits to each odour) and reliably to associate odours

156 with feeders of different types.

Training

To begin each experiment ants were allowed onto a $15 \mathrm{~cm}$ long, $1 \mathrm{~cm}$ wide runway, with a

drop of sucrose at the end. The first ant to encounter the sucrose was marked with a dot of paint, and all other ants were returned to the nest. The marked ant was allowed to drink to satiety and then return to the nest to unload the collected sugar. She was then allowed to make 7 further training visits to the runway and feeder. In each visit we recorded the number of pheromone depositions performed on the runway towards the feeder and towards the nest after foraging.

164 Over the 8 visits the quality and odour of the feeder was varied systematically so that the ant alternately encountered a moderate quality drop of sucrose solution $(0.55 \mathrm{M}$, 'safe') scented with one odour, or either a low $(0.1 \mathrm{M})$ or high $(1.0 \mathrm{M})($ 'risky') drop of sucrose scented with another odour. These values are clearly distinguishable by the ants (Wendt et al., 2018) and correspond to moderate, low, and high value food sources for L. niger (Detrain and Prieur, 2014). Note that the

169 average of the low and high quality solutions equals that of the moderate quality. The solutions were scented using either rosemary or lemon essential oils $(0.05 \mu \mathrm{l}$ per $\mathrm{ml})$. The runway leading to

171 the feeder was covered with a paper overlay scented identically to the sucrose solution being

172 offered. Overlays were scented by storing them in a sealed box containing cotton soaked in

173 essential oil. Overlays were discarded after each return to the nest, to ensure fresh odour and to

174 prevent a build-up of trail pheromone from occurring. 
175 Testing

After the 8 training visits, the runway was replaced with a Y-maze $($ arm length $10 \mathrm{~cm}$,

177 bifurcation angle $\left.120^{\circ}\right)$. The stem of the Y-maze was overlaid with unscented paper, whereas the

178 two other arms were covered with scented overlays - one bearing the 'risky' associated scent, and

179 the other the 'safe' associated scent. The maze tapered at the bifurcation to ensure that the ant

180 perceives both scented arms at the same time (following Czaczkes, 2018a). No sucrose was present

181 on the Y-maze. We recorded the ants' initial arm decision, defined by the ants' antennae crossing a

182 line $2 \mathrm{~cm}$ from the bifurcation point. We also recorded the ants' final decision, defined by the ant

crossing a line $8 \mathrm{~cm}$ from the bifurcation point. However, the initial and final decisions of the ants

184 were almost always the same, and analysis of either choice provides the same results (see ESM1).

For brevity we henceforth discuss only the initial decision data. On reaching the end of an arm the ant was allowed to walk onto a piece of paper and brought back to the start of the Y-maze stem, to be retested. The Y-maze test was thus repeated 3 times, to assess reliability of the ant choice. However, this handling may have caused some disruption (see ESM1) and repeated unrewarded trials affect motivation, so we conservatively analysed only the first Y-maze test. After testing, the ant was permanently removed from the colony. In total we tested 64 ants equally divided among 4 different colonies.

For each tested ant, one odour corresponded to the 'risky' feeder and one to the 'safe'

193 feeder. The association between odour and feeder type, the initial feeder type encountered, the

194 initial value of the 'risky' feeder, the side on which the 'risky' or 'safe' associated odours were 195 presented on the Y-maze test, and the scents associated with the 'risky' and 'safe' options were all balanced between ants. Performing treatments blind was attempted, but due to the clear negative

197 contrast effects shown by ants on encountering a low quality food source after better ones (Wendt 
bioRxiv preprint doi: https://doi.org/10.1101/620054; this version posted April 29, 2019. The copyright holder for this preprint (which was not certified by peer review) is the author/funder, who has granted bioRxiv a license to display the preprint in perpetuity. It is made available under aCC-BY-NC-ND 4.0 International license.

\section{Experiment 2-Risk preference between options of different absolute value}

2 was designed to test whether risk aversion would be maintained 'irrationally', that is, when the

'risky' feeder had a higher average quality than the 'safe' feeder.

As in experiment 1 , the 'safe' feeder always presented a medium quality drop $(0.55 \mathrm{M}$,

204 indistinguishable for the ants from the solution provided ad libitum to the colony). However, the

205 'risky' feeder alternated between a low quality reward (0.1M) and a very high quality reward $206(1.5 \mathrm{M})$. The average molarity of the risky feeder $(0.8 \mathrm{M})$ was thus higher than the average molarity of the safe one. L. niger foragers can distinguish between the three presented molarities (Wendt et al., 2018). Moreover, in a pilot experiment, we observed that when presented with three different molarities ants do learn all three molarities and their associated odours (see ESM1). Each ant was tested on the Y-maze 5 times, but as in experiment 1, only data from the first test was ultimately used (see ESM1). In total we tested 64 ants from 8 new colonies. Each condition (scent association,

212 feeder order, risky feeder order, scent side on the Y-maze) was balanced and equally distributed 213 among colonies.

\section{Experiment 3-Risk preference between psychophysically balanced options}

One hypothesis explaining the widespread risk aversion found in animals towards reward quantities arises from the psychophysics of perception: intensity is generally perceived logarithmically (Kacelnik and Bateson, 1996; Kacelnik and El Mouden, 2013; see introduction). It is thus the geometrical average between the two risky alternatives that may describe the perceived value. This hypothesis predicts that animals should be indifferent between a safe and a risky option, if the risky option balances the logarithmic differences between the low and high quality reward. In experiment 2, these were not balanced: the geometrical average of the risky feeder ( 
bioRxiv preprint doi: https://doi.org/10.1101/620054; this version posted April 29, 2019. The copyright holder for this preprint (which was not certified by peer review) is the author/funder, who has granted bioRxiv a license to display the preprint in perpetuity. It is made available under aCC-BY-NC-ND 4.0 International license.

223 risky option may still have been perceived as worse than the safe option. In this experiment we set

224 out to offer a 'risky' option in which the perceived qualities of the low and high reward were

225 balanced relative to the moderate reward. We chose a moderate reward of $0.3 \mathrm{M}$, and a low and

226 high reward of $0.1 \mathrm{M}$ and $0.9 \mathrm{M}$ respectively. The geometrical average of the risky option (

$227 \sqrt{0.1 \times 0.9}=0.3$ ) was now equal to the one of the safe option. We thus hypothesised that ants

228 would be indifferent between these two options. Each ant was tested on the Y-maze 5 times, but

229 again only data from the first test was used (see S1). In total we tested 40 ants from 10 different

230 colonies. Each condition (scent association, feeder order, risky feeder order, scent side in the Y-

231 maze) was balanced and equally distributed among colonies.

\title{
232 Statistical analysis
}

and Schielzeth (2011), we included in the models only factors and interactions for which we had $a$

priori reasons for including. We employed generalized linear mixed effect models using the package Ime4 (Bates et al., 2015), with colonies as a random effect. Y-maze choice data was

\author{
Initial decision $=$ \\ first presented feeder( risky-safe)* \\ first presented risky alternative ( good-bad) + \\ random effect ( colony)
}

modelled using a binomial distribution and logit link function. We used the following model:

We then used the package car (Fox and Weisberg, 2011) to test which factors of the model

239 had a significant effect on the dependent variable. Subsequently, we carried out post-hoc analysis

240 with Bonferroni correction using the package emmeans (Lenth, 2018) both for the general

241 preference of the ants for either the safe or the risky feeder (safe choice probability against

242 random probability), and for the factors with a significant effect to analyse the direction of the

243 difference. Plots were generated using the package ggplot2 (Wickham, 2009). 
bioRxiv preprint doi: https://doi.org/10.1101/620054; this version posted April 29, 2019. The copyright holder for this preprint (which was

not certified by peer review) is the author/funder, who has granted bioRxiv a license to display the preprint in perpetuity. It is made available under aCC-BY-NC-ND 4.0 International license.

Pheromone deposition count was modelled using a poisson distribution and logit link

245 function. Good model fit was confirmed using the DHARMa package (Hartig, 2018), and the pscl

246 package (Jackman, 2017; Zeileis et al., 2008) was used to produce the zero-inflated poisson models

247 when needed. Pheromone deposition was not the focus of the current study, but we include it as

248 descriptive data since it may shed light on how individual perception can shape group choice. We

249 modelled pheromone deposited towards the nest and pheromone deposited on the way back

250 separately, since these are conceptually very different: depositions towards the food reflect the

251 ants' expectation, and depositions on the return to the nest reflect the ants' perception. The

252 models used were the following:

Pheromone towards the drop =

visit (2-8)*

value (molarity)+

random effect ( ant nested in colony)

Pheromone back to the nest $=$

visit (1-8)*

value (molarity)+

Pheromone deposition data from each of the three experiments were analysed separately,

255 as they were taken by three separate experimenters, and so could not reliably be compared

256 between experiments. Path choice decisions allow much less observer error, so Y-maze data can be

257 pooled between experiments.

Only main results are reported below. For the full analysis see ESM2. The raw data for all

259 the experiments can be found in the supplemental materials ESM3. 


\section{Results}

261 Experiment 1 -Risk preference between options of equal absolute value

262 $279 z=2.616, p=0.036)$

\section{$\gamma$-maze choice tests}

\section{Pheromone deposition}

Ants were strongly risk averse, with $91 \%$ (58/64) ants initially choosing the safe option

(figure 1) (GLMM post-hoc with estimated means, probability=0.911, SE=0.36, $z=5.142, p<0.0001$ ).

We found no effect of the first presented feeder (GLMM Analysis of Deviance, Chi square=0.709,

$D F=1, p=0.3$ ), nor of the first presented risky alternative (Chi square=0, $D F=1, p=1$ ), nor of the interaction between those two factors (Chi square=0, DF=1, $p=1$ ).

Considering pheromone deposition towards the feeder, we found an effect of molarity (GLMM Analysis of Deviance, Chi square=12.992, DF=2, $p=0.001$ ) and an effect of the interaction between molarity and visit number (GLMM Analysis of Deviance, Chi square=14.469, DF=2, $p=0.0007)$. Specifically, we found that the ants deposited overall more pheromone when going towards the $0.55 \mathrm{M}$ drop in comparison to the $1.0 \mathrm{M}$ drop (figure $2 \mathrm{~A}$, GLMM post-hoc with estimated means, estimate $=0.657, \mathrm{SE}=0.227, \mathrm{z}=2.891, \mathrm{p}=0.015)$. Note that the ant may be expecting to find the $0.1 \mathrm{M}$ drop when going towards the $1.0 \mathrm{M}$, because it last experienced the low value associated with that scent. We found no differences in pheromone deposition between the other molarities. Overall, the ants deposited more pheromone on the way to the safe feeder relative to the risky one (GLMM post-hoc with estimated means, estimate $=0.498, S E=0.19$, 
Specifically, we found that the ants deposited overall less pheromone when going back from the

$0.1 \mathrm{M}$ drop in comparison to the $0.55 \mathrm{M}$ drop (figure 2D, GLMM post-hoc with estimated means,

estimate $=-2.67, \mathrm{SE}=0.154, \mathrm{z}=-17.352, \mathrm{p}<0.0001)$ and from the $0.1 \mathrm{M}$ drop in comparison to the

1.0M drop (GLMM post-hoc with estimated means, estimate=-2.78, SE=0.194, z=-14.308, $\mathrm{p}<0.0001)$. However, there was no difference between the $0.55 \mathrm{M}$ drop and the $1.0 \mathrm{M}$ drop. Overall the ants deposited more pheromone on the way back from the safe feeder relative to the risky one (GLMM post-hoc with estimated means, estimate=1.28, SE=0.14, z=9.149, $p<0.0001$ ).

Experiment 2 - Risk preference between options of different absolute value

\section{Y-maze choice tests}

Ants were again strongly risk averse, with $75 \%$ (48 / 64) ants initially choosing the safe

option (figure 1)(GLMM post-hoc with estimated means, probability=0.792, SE=0.068, z=3.248, $p=0.001$ ). We found no effect of the first presented feeder (GLMM Analysis of Deviance, Chi

square $=2.015, D F=1, p=0.156$ ), nor of the first presented risky alternative (Chi square=0.197, $D F=1$,

The data for the pheromone deposition are summarized in figure $2 \mathrm{~B}$ and $2 \mathrm{E}$.

(figure 2B, GLMM Analysis of Deviance, Chi square=7.489, DF=2, p=0.024). However, post-hoc

small that bonferroni correction in the post-hoc analysis brought them above significance. 
$306 \mathrm{DF}=2, \quad \mathrm{p}=0.003)$. Ants deposited less pheromone for the $0.1 \mathrm{M}$ drop in comparison to the $0.55 \mathrm{M}$

307 drop (figure 2E, GLMM post-hoc with estimated means, estimate=-2.683, SE=0.17, z=-15.742, $308 \mathrm{p}<0.0001$ ), less pheromone for the $0.1 \mathrm{M}$ in comparison to the $1.5 \mathrm{M}$ (GLMM post-hoc with 309 estimated means, estimate=-3.474, $S E=0.204, z=-17, p<0.0001$ ) and less for the $0.55 \mathrm{M}$ in comparison to the $1.5 \mathrm{M}$ (GLMM post-hoc with estimated means, estimate=-0.79, SE=0.19, z=$3114.144, p=0.0001)$. Overall the ants deposited more pheromone on the way back from the safe

312 feeder relative to the risky one (GLMM post-hoc with estimated means, estimate=0.946, SE=0.14, $313 z=6.341, p<0.0001)$.

314 Experiment 3-Risk preference between psychophysically-balanced options

\section{$315 \quad$ Y-maze choice tests}

(GLMM post-hoc with estimated means, probability=0.535, SE=0.086, z=0.403, $p=0.687$ ). square $=4.424, D F=1, p=0.0354)$. Specifically, $71 \%$ of the ants that were presented with the safe feeder in visit 1 choose the safe smell during testing, while $35 \%$ of the ones presented with the risky feeder first did.

\section{Pheromone deposition}

324 (GLMM, Chi square=16.133, $D F=2, p=0.0003$ ). Ants deposited more pheromone when going

325 towards the $0.3 \mathrm{M}$ drop in comparison to the $0.9 \mathrm{M}$ drop (figure $2 \mathrm{C}$, GLMM post-hoc with estimated 326 means, estimate $=10.444, S E=1.751, z=3.769, p=0.0007)$, while we found no difference between $3270.1 \mathrm{M}$ and $0.3 \mathrm{M}$ (GLMM post-hoc with estimated means, estimate=0.477, SE=0.174, z=-2.032, 
$329 \mathrm{SE}=3.452, \mathrm{z}=2.317, \mathrm{p}=0.082$ ). Overall, ants deposited more pheromone for the safe feeder (GLMM

330 post-hoc with estimated means, estimate $=4.679, \mathrm{SE}=1.751, \mathrm{z}=4.124, \mathrm{p}=0.0001$ )

332 (GLMM, Chi square=47.083, DF=2, $\quad \mathrm{p}<0.0001)$. Ants deposited less pheromone when returning

333 from the $0.1 \mathrm{M}$ drop in comparison to the $0.3 \mathrm{M}$ one (figure $2 \mathrm{~F}$, GLMM post-hoc with estimated

334 means, estimate $=-882, \mathrm{SE}=0.143, \mathrm{z}=-6144, \mathrm{p}<0.0001$ ), less for the $0.1 \mathrm{M}$ in comparison to the $0.9 \mathrm{M}$

335 (GLMM post-hoc with estimated means, estimate=-1.479, SE=0.18, $z=-8193, p<0.0001$ ) and less for

336 the $0.3 \mathrm{M}$ in comparison to the $0.9 \mathrm{M}$ (GLMM post-hoc with estimated means, estimate $=-0.597$,

$337 \mathrm{SE}=0.165, \mathrm{z}=-2.615, \mathrm{p}=0.001)$. Overall the ants deposited the same amount of pheromone on the

338 way back from the safe feeder relative to the risky one (GLMM post-hoc with estimated means,

339 estimate $=0.142, \mathrm{SE}=0.126, \mathrm{z}=1.134, \mathrm{p}=1)$. 
bioRxiv preprint doi: https://doi.org/10.1101/620054; this version posted April 29, 2019. The copyright holder for this preprint (which was not certified by peer review) is the author/funder, who has granted bioRxiv a license to display the preprint in perpetuity. It is made available under aCC-BY-NC-ND 4.0 International license.

340

342

343

344

345

346

347

348

349

\section{Discussion}

Ants show strong risk aversion given equal average payoffs between the risky and safe options (0.1/1.0M vs. $0.55 \mathrm{M}$, experiment 1$)$. Even if the risky option offers $45 \%$ higher mean payoffs than the safe reward $(0.1 \mathrm{M} / 1.5 \mathrm{M}$ vs. $0.55 \mathrm{M})$, ants still show strong risk aversion (experiment 2). We predicted, based on psychophysical principles, that logarithmically-balanced rewards should be perceived as having equal value. We tested this in a situation where the risky reward offered $66 \%$ higher payoffs than the safe reward $(0.1 / 0.9 \mathrm{M}$ vs $0.3 \mathrm{M})$ and observed, as predicted, indifference between the two options.

\section{Support for the perceptual basis of risk sensitivity}

Our demonstration of risk aversion in resource amounts strongly support the perceptual, descriptive theory of risk sensitivity proposed by Kacelnik \& Bateson (1996) and developed by Kacelnik \& El Mouden (2013). Specifically, our data suggest functional risk aversion arising from risk neutrality filtered through logarithmic perception. Budget Rule theories (Stephens, 1981) would also predict risk aversion in our context, since the ants are on a positive energy budget - Lasius niger would survive for over a week without feeding. However, our ability to accurately predict an indifference point based on logarithmic perception strongly implies that perceptual mechanisms are driving risk aversion in this species. Alternatively, we may have by chance chosen the precise point where logarithmic balancing matches the balance point between improved average gains from a risky option and the premium garnered by a safe bet according to the budget rule. However, this seems unlikely.

The ants in our experiments never showed a preference for the risky alternative. This may seem to imply that the ants were failing to learn the risky option, and associate it with an odour. However, this hypothesis can be ruled out, as it cannot account for the results of experiment 3, 
where neither food sources is preferred. If the ants were unable to learn the risky option, the only other explanation for experiment 3 would be that a $0.3 \mathrm{M}$ is not preferred over complete uncertainty. This can be ruled out, however, as ants clearly prefer $0.3 \mathrm{M}$ over $0.1 \mathrm{M}$ (ESM1).

The Budget Rule is neither supported nor refuted

the ants are on a positive energy budget - Lasius niger would survive for over a week without

371 feeding. However, our ability to accurately predict an indifference point based on logarithmic perception strongly implies that perceptual mechanisms are driving risk aversion in this species.

Our data neither supports nor refutes the Budget Rule (Caraco et al., 1980; Lim et al., 2015; would have behaved on a different energy budget. Lim et al. (2015) strongly critiques SUT, since it predicts suboptimal behaviour, which should be selected against. Logarithmic perception, however, is a widespread phenomenon in the animal kingdom, from roundworms (Luo et al., 2008) to humans (Fechner, 1860), and is argued that the logarithmic scale is the best possible neural representation of magnitudes among other biologically feasible scales (Portugal and Svaiter, 2011). A more precise food evaluation may require more energy than the energy gained from the additional precision. However, this has never been tested in the context of risk sensitivity (Lim et al., 2015). Even if the benefits accrued from a more linear perception of value would outweigh their costs, developmental constraints or pleiotropy may prevent such perception from evolving. 
the context of losses. This again derives from logarithmic perception of cumulative gains and

at zero. Rather, gains and losses are defined relative to a reference point, which is usually the

expected payoff, but may be socially induced (e.g. by comparing ones own salary to that of ones

colleagues). Anything above the reference point is perceived as a gain and anything below the

reference point is a loss. Disappointment for a lower value after a reference has been established

has already been demonstrated in the honeybee (Couvillon and Bitterman, 1984) and ants (Wendt

et al., 2018), and suggested in bumblebees (Wiegmann et al., 2003). The reference point for our

colonies might have been $0.5 \mathrm{M}$ : the solution that the ants are regularly fed on. If this were the

case, in experiment 1 the true choice would be between an always neutral value (0.55M, safe), and

a risk between a gain $(1.0 \mathrm{M})$ and a loss $(0.1 \mathrm{M})$. This hypothesis is also supported by the fact that

almost no pheromone was deposited for the 0.1M drop. In this case Prospect Theory would still

predict risk aversion, as losses are assumed to be perceived more strongly than gains. To test this

hypothesis we repeated experiment 1 , but with colonies that had been fed ad libitum $1.5 \mathrm{M}$

sucrose 1 month prior testing (data and procedure can be found in ESM1). If the ants were taking

their standard feeding solution as a reference point, every presented solution in this experiment

should have been perceived as a loss, and so the ants should have showed risk-seeking. However,

we observed the same preference that we saw in the main first experiment - strong risk aversion.

Either the ants behaviour is poorly described by Prospect Theory, or the normal feeding solution normal feeding solution, as the four-day food deprivation period may erase the ants memory of

410 the feeding solution. Instead, the reference point could be the most common solution in the

411 current context. In experiment 1 this would be $0.55 \mathrm{M}$, maintaining the same situation of one

412 neutral vs. a loss or a gain, and so predicting the same outcome under Prospect Theory. This

413 hypothesis, however, does not fit the result obtained in experiment 3: if the $0.3 \mathrm{M}$ would have been 
414 taken as a reference, we should still have observed a preference for the safe option. Either

415 Prospect Theory does not well describe the behaviour of ants, or their reference point remains at 0

416 in every situation, with every reward being a gain: in the domain of gains Prospect Theory predicts

417 simple logarithmic value perception.

Risk neutrality at the colony level

Does our understanding of individual behaviour in a risk-choice situation help explain the

421 risk indifference of ants at a colony levels (Hübner and Czaczkes, 2017)? Pheromone deposition

422 rates of individual foragers vary hugely between individuals, even when presented with identical

423 food sources. This is to be expected, given the fact that individual variability may aid collective

424 decisions (Dussutour et al., 2009; O'Shea-Wheller et al., 2017). However, the appropriate measure

425 of pheromone for colony-level decisions is total pheromone deposited. Examining the mean

426 deposition rates for both feeders in experiment 1, we see that ants, on average, deposited more

427 pheromone to the safe feeder (5.5 dots per ant) than the risky feeder (3.9 dots per ants). In

428 Hübner \& Czaczkes (2017) each ant made only one or two visits to the feeder, but even when

429 considering only the first two visits ants made more pheromone depositions to the safe (1.5 dots

430 per ant) than to the risky ( 0.89 dots per ant) feeder. The finding of risk neutrality at the colony level

431 is thus still a puzzle. However, the two experiments are not directly comparable. Firstly, in the

432 current experiment pheromone was removed from the trail every visit. Pheromone presence is

433 known to reduce further pheromone deposition (Czaczkes et al., 2013), perhaps damping out the

434 differences between the two feeders. Secondly, the presence of odours on a path affects

435 pheromone deposition: while pheromone deposition on odourless paths is usually higher on the

436 nestward journey (Beckers et al., 1993; Czaczkes and Heinze, 2015; Czaczkes et al., 2013, 2016),

437 pheromone deposition is higher on outward journeys on scented paths (this study, Czaczkes et al.,

$4382018 b, 2018 c)$. Finally, it should be noted that perception of pheromone, much like perception of 
439 quality, is also logarithmic (von Thienen et al., 2014), thus emphasising initial differences in

440 pheromone concentration but damping out differences between strong trails. Nevertheless, it

441 seems that colony-level decision-making effectively filters out the ants individual perceptual

442 constrains (this study, Sasaki and Pratt, 2011), but the mechanism used to achieve this is still

443 unknown.

444 In this study, we found that ants demonstrate risk aversion due to a logarithmic perception 445 of food value. Individual risk preference does not predict colony behaviour, which seems able to 446 filter out perceptual biases. 


\section{References}

Banschbach, VS, Waddington, KD. 1994. Risk-sensitive foraging in honey bees: no consensus among individuals and no effect of colony honey stores. Anim. Behav. 47, 933-941.

Bates, D, Mächler, M, Bolker, B, Walker, S. 2015. Fitting Linear Mixed-Effects Models Using $\{$ Ime4\}. J. Stat. Softw. 67, 1-48.

Becker, GM, Degroot, MH, Marschak, J. 1964. Measuring utility by a single-response sequential method. Behav. Sci. 9, 226-232.

Beckers, R, Deneubourg, JL, Goss, S, Pasteels, JM. 1990. Collective decision making through food recruitment. Insectes Sociaux 37, 258-267.

Beckers, R, Deneubourg, JL, Goss, S. 1993. Modulation of trail laying in the ant Lasius niger Hymenoptera: Formicidae and its role in the collective selection of a food source. J. Insect Behav. 6, 751-759.

Boomsma, JJ, Gawne, R. 2018. Superorganismality and caste differentiation as points of no return: how the major evolutionary transitions were lost in translation. Biol. Rev. 93, 28-54.

Burns, DDR, Sendova-Franks, AB, Franks, NR. 2016. The effect of social information on the collective choices of ant colonies. Behav. Ecol. 27, 1033-1040.

Caraco, T, Martindale, S, Whittam, TS. 1980. An empirical demonstration of risk-sensitive foraging preferences. Anim. Behav. 28, 820-830.

Cartar, RV. 1991. A Test of Risk-Sensitive Foraging in Wild Bumble Bees. Ecology 72, 888895.

Cartar, RV, Dill, LM. 1990. Why are bumble bees risk-sensitive foragers? Behav. Ecol. Sociobiol. 26, 121-127.

Couvillon, PA, Bitterman, ME. 1984. The overlearning-extinction effect and successive negative contrast in honeybees Apis mellifera. J. Comp. Psychol. Wash. DC 1983 98, 100-109.

Czaczkes, TJ. 2018a. Using T- and Y-mazes in myrmecology and elsewhere: a practical guide. Insectes Sociaux 65, 213-224.

Czaczkes, TJ, Heinze, J. 2015. Ants adjust their pheromone deposition to a changing environment and their probability of making errors. Proc. Biol. Sci. 282. 
Czaczkes, TJ, Grüter, C, Ellis, L, Wood, E, Ratnieks, FLW. 2013. Ant foraging on complex

trails: route learning and the role of trail pheromones in Lasius niger. J. Exp. Biol. 216, 188-197.

Their Role in Social Insect Colony Organization. Annu. Rev. Entomol. 60, 581-599.

Czaczkes, TJ, Brandstetter, B, di Stefano, I, Heinze, J. 2018b. Greater effort increases perceived value in an invertebrate. J. Comp. Psychol. Wash. DC 1983 132, 200-209.

Czaczkes, TJ, Koch, A, Fröber, K, Dreisbach, G. 2018c. Voluntary switching in an invertebrate: The effect of cue and reward change. J. Exp. Psychol. Anim. Learn. Cogn. 44, 247-257. $1763-1767$.

Detrain, C, Deneubourg, JL. 2008. Collective Decision-Making and Foraging Patterns in niger to sugar resources. J. Insect Physiol. 64, 74-80. spatio-temporal changes in aphid colonies Cinara cedri. Dong Wu Xue Bao 51, 161-166.

Ants and Honeybees. In Advances in Insect Physiology, Elsevier, pp. 123-173.

environment: persistence and reward in the foraging decisions of bumblebees. Interface Focus 7 , 20160149.

Dussutour, A, Beekman, M, Nicolis, SC, Meyer, B. 2009. Noise improves collective decision-making by ants in dynamic environments. Proc. R. Soc. B Biol. Sci. 276, 4353-4361. 
Fechner, GT. 1860. Elemente der psychophysik Leipzig : Breitkopf und Härtel.

Forstmeier, W, Schielzeth, H. 2011. Cryptic multiple hypotheses testing in linear models:

Overestimated effect sizes and the winner's curse. Behav. Ecol. Sociobiol. 65, 47-55.

Fox, J, Weisberg, S. 2011. An R Companion to Applied Regression. Thousand Oaks, CA:

Sage.

Fülöp, A, Menzel, R. 2000. Risk-indifferent foraging behaviour in honeybees. Anim. Behav.

$60,657-666$.

Erlbaum.

Regression Models.

Strangeness of Insect Societies W.W. Norton.

Hübner, C, Czaczkes, TJ. 2017. Risk preference during collective decision making: ant colonies make risk-indifferent collective choices. Anim. Behav. 132, 21-28.

Hurly, AT. 2003. The twin threshold model: risk-intermediate foraging by rufous hummingbirds, Selasphorus rufus. Anim. Behav. 66, 751-761.

Jackman, S. 2017. pscl: Classes and Methods for R Developed in the Political Science

Computational Laboratory Sydney, New South Wales, Australia: United States Studies Centre, University of Sydney.

Kacelnik, A, Bateson, M. 1996. Risky Theories-The Effects of Variance on Foraging 528 Decisions. Integr. Comp. Biol. 36, 402-434. 
Lim, IS, Wittek, P, Parkinson, J. 2015. On the origin of risk sensitivity: the energy budget

rule revisited. Anim. Behav. 110, 69-77.

Luo, L, Gabel, CV, Ha, HI, Zhang, Y, Samuel, ADT. 2008. Olfactory Behavior of Swimming $C$.

elegans Analyzed by Measuring Motile Responses to Temporal Variations of Odorants. J.

Neurophysiol. 99, 2617-2625.

Mayack, C., Naug, D. 2011. A changing but not an absolute energy budget dictates risksensitive behaviour in the honeybee. Anim. Behav. 82, 595-600.

Mehlhorn, K, Newell, BR, Todd, PM, Lee, MD, Morgan, K, Braithwaite, VA, Hausmann, D,

Fiedler, K, Gonzalez, C. 2015. Unpacking the exploration-exploitation tradeoff: A synthesis of human and animal literatures. Decision 2, 191-215.

Oberhauser, FB, Koch, A, Czaczkes, TJ. 2018. Small differences in learning speed for different food qualities can drive efficient collective foraging in ant colonies. Behav. Ecol. Sociobiol. $72,1096$.

O'Shea-Wheller, TA, Masuda, N, Sendova-Franks, AB, Franks, NR. 2017. Variability in individual assessment behaviour and its implications for collective decision-making. Proc R Soc B 284, 20162237.

Perez, SM, Waddington, KD. 1996. Carpenter Bee Xylocopa micans Risk Indifference and a

Review of Nectarivore Risk-Sensitivity Studies. Am. Zool. 36, 435-446.

Scale. Minds Mach. 21, 73-81.

Price, RI, Grüter, C, Hughes, WOH, Evison, SEF. 2016. Symmetry breaking in massrecruiting ants: extent of foraging biases depends on resource quality. Behav. Ecol. Sociobiol. 70, $1813-1820$. and Tests. Q. Rev. Biol. 52, 137-154.

R Core Team. 2017. R: A Language and Environment for Statistical Computing Vienna, multiattribute decision making in non-neuronal organisms. Anim. Behav. 100, 44-50. 
Reid, CR, MacDonald, H, Mann, RP, Marshall, JAR, Latty, T, Garnier, S. 2016. Decision-

making without a brain: how an amoeboid organism solves the two-armed bandit. J. R. Soc.

Sasaki, T, Pratt, SC. 2011. Emergence of group rationality from irrational individuals.

Behav. Ecol. 22, 276-281.

Shafir, S. 2000. Risk-sensitive foraging: the effect of relative variability. Oikos $88,663-669$.

Shafir, S, Wiegmann, DD, Smith, BH, Real, LA. 1999. Risk-sensitive foraging: choice 1061.

Shapiro, MS. 2000. Quantitative analysis of risk sensitivity in honeybees Apis mellifera with variability in concentration and amount of reward. J. Exp. Psychol. Anim. Behav. Process. 26, $574 \quad 196-205$.

Stephens, DW. 1981. The logic of risk-sensitive foraging preferences. Anim. Behav. 29, $628-629$.

Stevens, SS. 2017. Psychophysics: Introduction to Its Perceptual, Neural and Social Prospects (Routledge).

von Thienen, W, Metzler, D, Choe, DH., Witte, V. 2014. Pheromone communication in ants: a detailed analysis of concentration-dependent decisions in three species. Behav. Ecol. Sociobiol. 68, 1611-1627.

Tuzlukov, VP. 2013. Signal Detection Theory Springer Science \& Business Media.

Wendt, S, Strunk, KS, Heinze, J, Roider, A, Czaczkes, TJ. 2018. Relative value perception in an insect: positive and negative incentive contrasts in ants. BioRxiv 330241. (under revision for eLife)

Wickham, H. 2009. ggplot2: Elegant Graphics for Data Analysis Springer-Verlag New York. 
bioRxiv preprint doi: https://doi.org/10.1101/620054; this version posted April 29, 2019. The copyright holder for this preprint (which was

not certified by peer review) is the author/funder, who has granted bioRxiv a license to display the preprint in perpetuity. It is made available under aCC-BY-NC-ND 4.0 International license.

Zeileis, A, Kleiber, C, Jackman, S. 2008. Regression Models for Count Data in R. J. Stat.

592 Softw. 27., 1611-1627. doi:10.1007/s00265-014-1770-3 
bioRxiv preprint doi: https://doi.org/10.1101/620054; this version posted April 29, 2019. The copyright holder for this preprint (which was

not certified by peer review) is the author/funder, who has granted bioRxiv a license to display the preprint in perpetuity. It is made available under aCC-BY-NC-ND 4.0 International license.

\section{Figure legends}

594 Figure 1: Proportion of ants choosing the safe feeder. Ants preference is different from chance level in experiment 1

595 (prob. $=0.911, S E=0.36, z$ ratio $=5.142, p$-value $<0.0001)$ and in experiment 2 (prob. $=0.792, S E=0.068, z$ ratio $=3.248, p$ -

596 value $=0.001$ ), but not in experiment 3 (prob. $=0.535, S E=0.086, \mathrm{z}$ ratio=0.403, $p$-value=0.687).

597 Figure 2: Amount of pheromone deposited by the ants going to the drop and back to the nest across visits in the three

598 experiments. Considering the pheromone deposited on the way to the drop, we found a higher deposition rate for the

599 safe feeder in experiment 1 (A) and in experiment 3 (C) but not in experiment 2 (B). Considering the pheromone

600 deposited on the way back to the nest, we found a higher deposition rate for the safe alternative in experiment 1 (D)

601 and experiment $2(E)$, but not in experiment $3(F)$.

602 
bioRxiv preprint doi: https://doi.org/10.1101/620054; this version posted April 29, 2019. The copyright holder for this preprint (which was

not certified by peer review) is the author/funder, who has granted bioRxiv a license to display the preprint in perpetuity. It is made available under aCC-BY-NC-ND 4.0 International license.

\section{Figures}

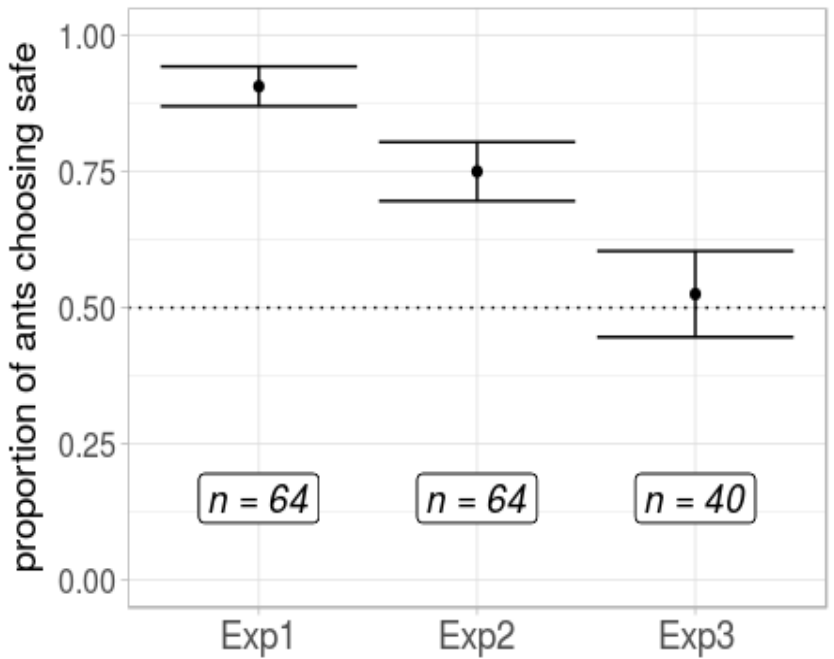

604

Safe: 0.55 Safe: $0.55 \quad$ Safe: 0.3

Risky: 0.1/1.0 Risky: 0.1/1.5 Risky: 0.1/0.9
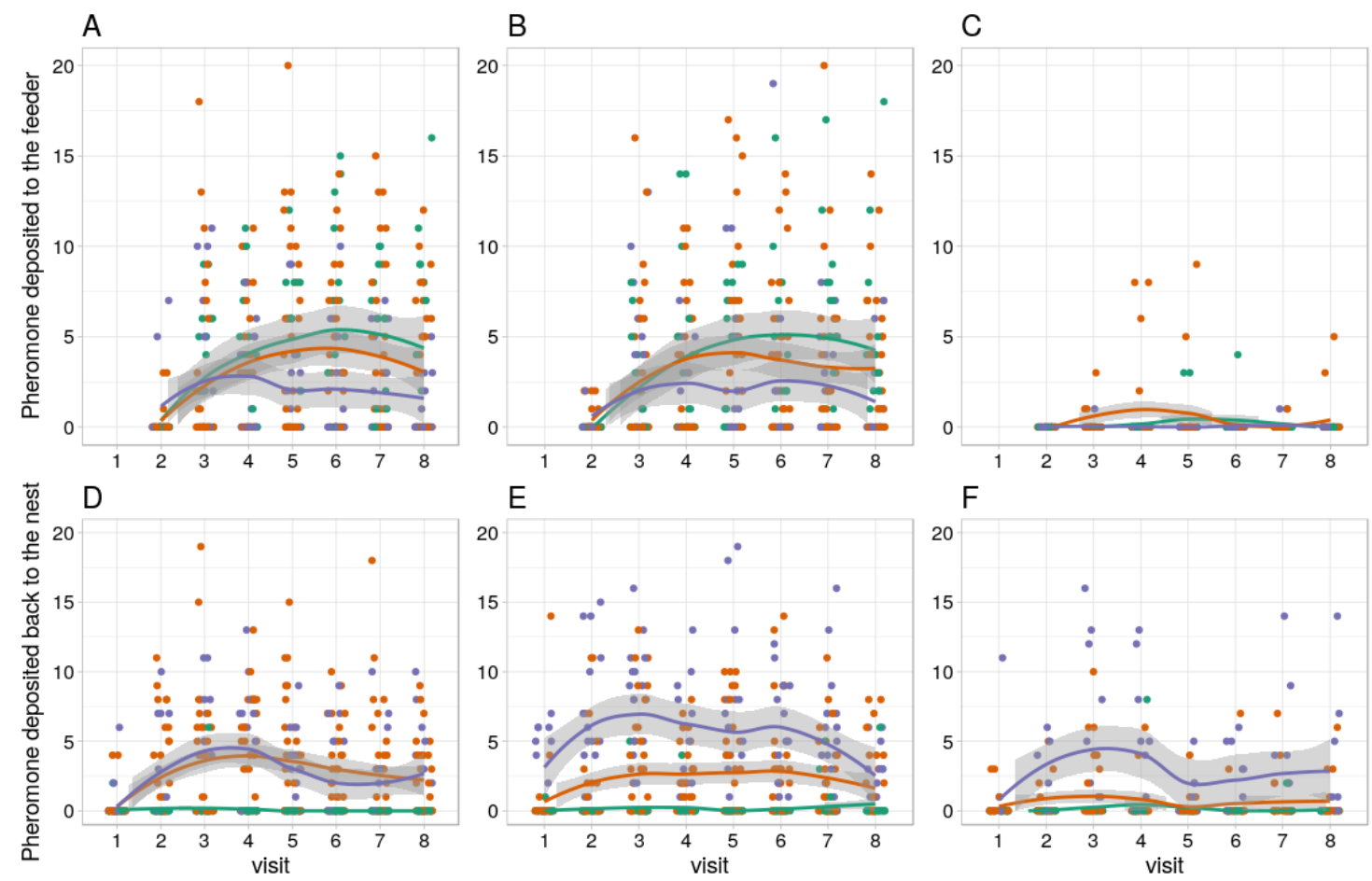

molarity $\rightarrow 0.1 \rightarrow 0.3 \rightarrow 0.9$ 